\title{
La Creatividad Artística en Tiempos de Pandemia
}

\author{
Artistic Creativity in Times of Pandemic
}

\section{Criatividade artística em tempos de pandemia}

\author{
Slink Víctor Dueñas Silva \\ sduenass@unsa.edu.pe \\ https://orcid.org/0000-0002-5552-8449 \\ Universidad Nacional de San Agustin de Arequipa, Arequipa - Perú
}

Recibido 02 de Diciembre 2021 | Arbitrado y aceptado 14 de Marzo 2022 | Publicado en 02 Junio 2022

\section{Resumen}

La creatividad es entendida como aquella capacidad que durante toda la historia del ser humano le ha permitido a este crecer, desarrollarse, en general, progresar de forma innovadora y eficiente incluso en momentos de gran dificultad donde los individuos se han visto obligados a lidiar con fenómenos naturales, desastres, etc. En ese sentido, desde diciembre de 2019 cuando se desató el virus posteriormente denominado Covid-19, el mundo comenzó a ver cambios en sus estilos de vida. Y, más tarde, cuando se decretó que este mal se había convertido en una pandemia mundial, se vieron afectados no solo las personas relacionadas a sectores de salud sino además económicos, sociales, políticos y también aquellos en el sector artístico-cultural. Por todo ello, el presente artículo de investigación se propuso reflexionar sobre los efectos que ha tenido en la creatividad artística el complejo momento que se vive actualmente caracterizado principalmente por la pandemia de Covid-19 y poder comprender cómo ha servido esta para hacerle frente.

Palabras clave: Creatividad, creatividad artística, pandemia, Covid-19. 


\title{
ECNO \\ HUMANTSMO
}

\begin{abstract}
Creativity is understood as that capacity that throughout the history of the human being has allowed this to grow, develop, in general, progress in an innovative and efficient way even in moments of great difficulty where individuals have been forced to deal with phenomena natural disasters, etc. In that sense, since December 2019 when the virus later called Covid-19 was unleashed, the world began to see changes in their lifestyles. And, later, when it was decreed that this evil had become a global pandemic, not only people related to health sectors but also economic, social, political and also those in the artistic-cultural sector were affected. For all these reasons, this research article set out to reflect on the effects that the complex moment currently being experienced mainly characterized by the Covid-19 pandemic has had on artistic creativity and to be able to understand how this has served to cope with it.
\end{abstract}

Keywords: Creativity, artistic creativity, pandemic, Covid-19.

\section{Introducción}

Desde siempre, los seres humanos han recurrido a la creatividad como un recurso para transformar los espacios que los rodeaban, adaptarse a la sociedad y avanzar en ella. La creatividad está adherida a la historia humana y sus acciones representan aportes, tanto cotidianos como complejos, para la supervivencia y desarrollo así como la mejora en la calidad de vida de las personas (Campos y Palacios, 2018).

Hablar pues de creatividad es hablar de potencial humano y de transformación social, es hablar de modelos de sociedad que tengan iniciativa y capacidad para resolver los problemas, es, en definitiva, hablar de progreso (Fernández et al. 2019)

En ese sentido, la humanidad a través de milenios se ha tenido que enfrentar a innumerables obstáculos masivos como desastres naturales, desastres producidos por los propios seres humanos, plagas y enfermedades (Martínez-Taboas, 2020) y recientemente a una pandemia mundial ocasionada por la Covid-19 (Ferrer, 2020).

Es en este tipo de contextos que la creatividad se revela como una herramienta clave para que los sujetos sean capaces de resolver las complejidades que se presenten como parte del panorama social (Campos y Palacios, 2018). 


\section{HUMANISMO
ECNO}

Por todo lo expuesto, se pretende con la presente investigación reflexionar sobre los efectos que ha tenido en la creatividad artística el complejo momento que se vive en la actualidad, caracterizado principalmente por la pandemia de Covid-19.

\section{Metodología}

La búsqueda de la información para la revisión de literatura se hizo consultando las siguientes bases de datos como son Proquest, Scopus y Google Scholar para encontrar revistas del más alto nivel científico. Asimismo, cabe mencionar que para la realización de lo mencionado se emplearon también palabras clave como como Creatividad, Creatividad artística, Pandemia, Covid-19, Confinamiento.

Cada uno de estos descriptores se combinaron entre sí durante la búsqueda utilizando los operadores boléanos “and" y "or". Además, algunos de los criterios o filtros que se utilizaron para hacer muchos más específica la búsqueda y ser más precisos con los documentos encontrados en las diferentes bases de datos consultadas fueron: que sean artículos de revistas, y la antigüedad de la publicación que no sea mayor a 5 años.

Toda la información previa relacionada a los filtros aplicados y las especificaciones sirvieron como criterios de inclusión, es decir que sean artículos publicados en revistas científicas, que no excedan los cinco años de antigüedad, que la temática guarde relación con las variables de creatividad artística y tiempos de pandemia. Se excluyeron, en tanto, todos los documentos que no respetaran lo postulado así como aquellos que estaban incompletos o que tenían enlaces averiados.

\section{Resultados y Discusión}

\subsection{Creatividad}

\subsubsection{Nociones básicas}

Llanos (2020) citando a Vigotsky (2003) precisa que, dentro de todas las concepciones que se tienen de la creatividad es clave enfatizar que esta, por sobre todas las cosas, "es una fuerza necesaria para la existencia” (p. 137). 


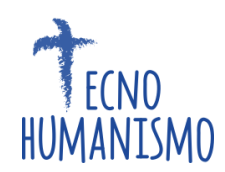

Sin embargo, la anterior es solo una adhesión de las múltiples perspectivas que intentan explicar qué es la creatividad. Campos y Palacios (2018) explican que no hay consenso para una definición única, y ello se debe a que se trata de un concepto adaptable, multifacético, dinámico y no cerrado al cambio. Al respecto, cabe mencionar que las diversas definiciones pueden estar comprimidas en dos corrientes o perspectivas opuestas: la clásica y la moderna (Elliot, 1991; Morales, 2001; Campos y Palacios, 2018).

La posición clásica de la creatividad se basa en una visión absorta en mitos y, lamentablemente, donde se fundamentan muchas falsedades que rodean este término. Así, era entendida la creatividad como un don que poseían unos cuantos individuos por talento y peculiaridad; en resumen, la creatividad, según esta perspectiva, es una cosa innata, determinada por la capacidad personal y resultado de un ejercicio no aprendido (Romero, 2010 y Caerols, 2013, citados en Campos y Palacios, 2018)

De lado opuesto, los investigadores citan a Klimenko (2008) y Valqui (2009) para señalar que la creatividad desde la perspectiva actual y moderna está basada en una serie de procesos cognitivos ordinarios y comunes que realizan todas las personas, los mismos que pueden ser perfeccionados mediante el esfuerzo y la experiencia.

En ese sentido, la creatividad es una capacidad que todos los seres humanos tienen en menor o mayor medida (Fernández et al. 2019; Llanos, 2020). Estos autores agregan además, citando a Elisondo y Donolo (2010) y Krumm y Lemos (2012), que esta despegará en la medida que tenga un entorno favorable, así se aprecia la importancia del ambiente creativo para aprovechar y desarrollar todo el potencial creativo de una persona. Teniendo esa mirada social, se hacen importantes elementos como la acción social, socialización, percepción social, control social, formas de la influencia social, y todo tipo de factores interpersonales.

\subsubsection{Elementos de la creatividad}

Rhodes (1961) citado en Liu \& Chang (2017) señala que la creatividad se fundamenta en el uso articulado de cuatro componentes: persona, proceso, prensa y producción, de ahí surge la denominación "Modelo de las 4Ps". En ese sentido, el funcionamiento de estos elementos inicia con la persona que estimulada por la prensa (o entorno) anima un proceso para la realización de un producto. 
Campos y Palacios (2018) se basan en este modelo y presentan a los elementos de la siguiente forma:

- El sujeto creativo

Este, a su vez, se puede dividir basado en dos enfoques, el personológico y el sociocultural. El primero de ellos, se vincula a la persona de forma individual, resaltando una serie de características psicológicas que son parte de la singularidad de cada sujeto.

En el otro extremo, el enfoque sociocultural señala que la creatividad no debe tomar en cuenta únicamente los rasgos personales del sujeto, sino además, la interacción de este con su contexto. Así, el creativo así como su creación se ven influenciados por aspectos externos, por lo que no debe ignorarse nunca el entorno social.

- El ambiente

La forma en la que una persona interactúa con su contexto social, entiéndase por este los espacios compartidos con la familia, escuela y sociedad en general, van a ser determinantes en la importancia que se le dé a la creatividad en el desempeño de cualquier función: artística, laboral, académica, personal, etc.

\section{- El proceso creativo}

Se refiere al enlace entre el inicio del acto creativo, que surge del interior del sujeto (y las influencias externas) y la culminación de la acción creativa en la elaboración de un producto.

\section{- Producto}

Se refiere a los objetos físicos, ideas, sistemas, servicios que dieron resultado de los procesos creativos.

De esta forma, al desagregar la creatividad con el modelo de las $4 \mathrm{ps}$ se crea una estructura que sirve para analizar y ordenar el conocimiento de esta capacidad. 


\subsubsection{Proceso creativo}

El modelo básico consiste en cuatro etapas descritas por Wallas (1926):

1. Preparación: Identificación y definición del problema, así como la recolección de información. Es un proceso consciente que depende de las habilidades analíticas y la educación del individuo.

2. Incubación: Asociación inconsciente de la información recopilada en la etapa anterior, se presenta cuando se abandona temporalmente el trabajo en el problema.

3. Iluminación: Momento en que una idea (solo una) que resuelve el problema llega repentinamente a la mente.

4. Verificación: Trabajo consciente de evaluación, refinamiento y desarrollo de la idea.

No obstante, López (2015) señala que además de las etapas anteriores, hay una fase previa: Calentamiento, que corresponde a la creación de un estado mental favorable hacia la creatividad e imaginación. Finalmente, en Campos (2017) se recogen todos los aspectos señalados previamente y se agrega una fase final: Elaboración, que es el momento exacto en que el proceso creativo da paso al producto y donde también se considera la etapa de comunicación del resultado final al público (Campos y Palacios, 2018).

Figura 1. Etapas del proceso creativo.

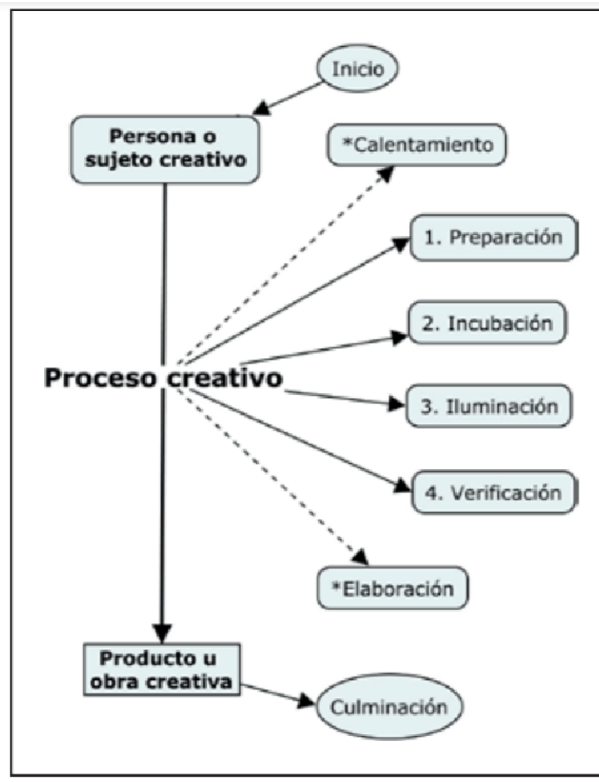

Fuente: Campos y Palacios (2018). 


\section{ECNO \\ HUMANISMO}

\subsubsection{Creatividad Artística}

Para llegar a la creatividad artística es importante recordar que esta se mueve alrededor del concepto de arte, el mismo que constituye una de las producciones, realizaciones o expresiones representacionales a través de las que todo ser humano muestra, de manera simbólica o representativa, la percepción de su realidad externa, de su realidad interna y de sus propias vivencias como persona (Sánchez, 2017).

En ese sentido, García y Morales (2011) citados en Fernández et al. (2019) indican que la creatividad puede abordarse, como se ha visto, desde múltiples y diferentes enfoques, una de ellas es su base artística. Con ello, la creatividad es presentada como un elemento indispensable en la ejecución artística.

Sánchez (2017) respalda lo anterior y señala que el arte como producción o realización humana está íntimamente ligado a la creatividad. La creatividad artística le va a permitir a todo individuo lograr producciones originales o novedosas, manifestadas de múltiples formas, llámese creatividad artístico plástica, creatividad musical, creatividad literaria, creatividad escénica, creatividad plástico-motora o quinestésica. Y, entre las formas más complejas de creatividad, se da en la creatividad publicitaria y en la arquitectónica, en la creatividad cinematográfica y en la creatividad profesional.

\subsection{Pandemia mundial}

\subsubsection{Covid-19}

En diciembre de 2019 se reportaron una serie de casos de pacientes hospitalizados con una enfermedad nueva caracterizada por neumonía e insuficiencia respiratoria, a causa de un nuevo coronavirus (SARS-CoV-2), en la provincia de Hubei, China. Posterior a ello, en febrero de 2020 la Organización Mundial de la Salud (OMS) ya había nombrado a este agente etiológico como COVID-19 (Coronavirus Disease, 2019) (Ferrer, 2020).

Pese a las medidas de contención, la fuente previa indica que la enfermedad traspasó fronteras y siguió avanzando hasta afectar al resto de los países de Asia, Medio Oriente y Europa. Fue así como el 11 de marzo, la OMS declaró como pandemia mundial a la COVID19 en una rueda de prensa en todo el globo que dirigió Tedros Ghebreyesus, director general de la entidad. 


\section{IECNO}

Esta enfermedad trajo consigo no solo millones de muertes alrededor del mundo sino consecuencias indirectas pero relacionadas con otros temas. Ello se debe a que las pandemias están caracterizadas por la confusión, temores, incertidumbre y la probabilidad de muertes en seres queridos. Asimismo, se relacionan con innumerables estresores sociales tales como hacer ajustes en las rutinas, separación de amigos y familiares, pérdida de empleos y aislamiento social (Martínez-Taboas, 2020). Esto, por supuesto, no deja de tener efectos en el ámbito cultural de la sociedad, donde residen las diversas expresiones artísticas previamente mencionadas, sino además en el mismo individuo y sus procesos creativos.

\subsection{Creatividad durante la pandemia}

La actual crisis sanitaria, económica y social en todo el mundo está teniendo enormes efectos en el sector creativo-artístico. El COVID-19 ha afectado muy gravemente a la vida cultural de las comunidades de las diversas esferas de arte (tanto en la creación, producción, distribución y acceso) y ha debilitado considerablemente la situación profesional, social y económica de los artistas y profesionales de la cultura. Asimismo, Las medidas de confinamiento también impactan directamente la capacidad de cada individuo para crear y distribuir nuevas expresiones artísticas y contenido cultural en el entorno digital, reduciendo la diversidad de las expresiones culturales en todo el mundo (Unesco, 2020; UCLG, 2020; Cárdenas-Pérez, 2021).

En ese sentido, los obstáculos en la creatividad artística se pueden dividir en dos perspectivas: aquellos efectos que se ha tenido en empresarios o empresas que han debido replantear sus estrategias de negocios, marcas, etc., hacia el mercado (es decir, hacia el exterior); como aquellos efectos producidos en el creativo, en la persona, de tal forma que se ha visto forzado a cambiar su proceso creativo, moldearlo, adecuarlo, etc., con este nuevo y complejo estímulo que es el confinamiento (es decir, internamente).

Como se ha visto, la creatividad es precisamente la capacidad de gestar soluciones alternas. Así, según narra la Unesco (2020) muchos artistas, gestores y profesionales de la cultura se han visto forzados a crear soluciones innovadoras y creativas, en la mayoría de los casos mediante el uso de herramientas digitales, para continuar sus actividades y conectarse con el público. 


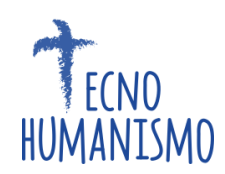

La música y el baile, accesibles en diversas plataformas digitales por ejemplo, han permitido a las personas expresarse y mantener vivos los lazos sociales en medio de las restricciones de movilidad y necesaria cuarentena. Este tiempo extraordinario también ha demostrado el papel central que juega la cultura en la construcción de la resiliencia y la cohesión social, se ha visto la solidaridad de muchos museos y galerías que han compartido su material digitalmente, incluso innovando con tours virtuales, entre otras herramientas.

Asimismo, si se estudia desde la perspectiva del sujeto, Gutiérrez (2020) precisa que el 'encierro', como muchos le llaman, ha sacado, en más de una ocasión, lo peor de los seres humanos. Ello se debe a que tras un tiempo sin poder salir físicamente, las emociones, como la tristeza, el miedo y el enojo, también se encierran y se reprimen dentro del ser, para luego salir en pequeñas explosiones que se manifiestan a través de cambios drásticos en los ciclos de sueño, hábitos alimenticios y en las maneras de interrelacionarnos con nuestros seres cercanos.

Por lo tanto, una salida para ser capaces de autorregularse y mantener la salud mental en su punto óptimo es recurrir a la expresión artística como medio para canalizar las emociones de una forma moderada, sana y productiva.

El autor también indicó que si bien muchos artistas se han visto en la necesidad de alterar sus procesos creativos, en la medida que estos se han ralentizado, cambiado, o se han visto influenciados por otros sentimientos y emociones que han servido para nutrir sus obras; cabe recalcar que el proceso del confinamiento ha servido, sobre todo, para masificar el arte así como democratizar la creatividad.

Así, se ha puesto en evidencia que el arte no pertenece a contextos delimitados como escenarios, museos, galerías, sino que puede mutar a ciberespacios, espacios públicos, etc., a la vez que se muestra que no es un concepto que "nace" con una minoría de prodigios, sino que si se promueve y fomenta, pertenece a cada uno de los seres humanos.

\section{Conclusiones}

El presente artículo ha reflexionado sobre los efectos que ha tenido en la creatividad artística la pandemia ocasionada por la Covid-19. En ese sentido, se ha presentado que este concepto, diverso y multifacético, a lo largo de toda la historia humana ha servido como una gran herramienta para sobrellevar problemas y enfrentar fenómenos complejos. Por ende, no es 


\section{HUMANISMO
HUMANO}

la excepción que durante la pandemia y sobre todo durante los meses de confinamiento obligatorio, la creatividad se ha manifestado tanto para empresarios y empresas que han debido alterar sus estrategias de marca y llegada al consumidor, como del otro lado desde la perspectiva del creador quien ha tenido que cambiar igualmente su proceso creador para adaptarse al nuevo contexto; así como también cabe mencionar la importancia que ha tenido la pandemia en aquellos que creían que no tenían o tenían poco desarrollada la capacidad de la creatividad para precisamente explotarla y demostrar que esta se encuentra cada vez más democratizada.

\section{Referencias}

Caerols, R. (2013). La creatividad, el mito del genio y la educación del talento a través de la obra de Woody Allen. Revista Electrónica de Investigación, Docencia y Creatividad, (2), $27-45$.

Campos, G. y Palacios, A. (2018) La creatividad y sus componentes. Creatividad y Sociedad (27) 167-183. http://creatividadysociedad.com/articulos/27/7.La creatividad y sus componentes.pdf

Cárdenas-Pérez, R. (2021) Emergencia del arte digital en la educación artística y las artes visuales en tiempos de pandemia. Pensamiento, palabra y obra. https://revistas.pedagogica.edu.co/index.php/revistafba/article/view/13066/9104

Elliott, J. (1991) Action research for educational change. Milton Keynes, Open University Press.

Elisondo, R. y Donolo, D. (2010). ¿Creatividad o inteligencia? That is not the question. Anales de Psicología, 26(2), 220-22.

Fernández, J., Llamas, F. y Gutiérrez-Ortega, M. (2019). Creatividad: Revisión del concepto. REIDOCREA, 8, 467-483.

Ferrer, R. (2020) Pandemia por COVID-19: el mayor reto de la historia del intensivismo. Medicina Intensiva, 44(6), 323-324.

García, F. y Morales, J. (2011). El impacto de la creatividad en la valoración artística. Arte, Individuo y Sociedad, 23(2), 69-84. 
Gutiérrez, G. (2020) La creatividad en el confinamiento: ¿Está cambiando la forma en cómo valoramos el arte? La Antigua, № 84, pp. 78 -79. ISSN 1010-8483.

Klimenko, O. (2008). La creatividad como un desafío para la educación del siglo XXI. Educación y Educadores, 11(2), 191-210.

Krumm, G. y Lemos, V. (2012). Actividades artísticas y creatividad en niños escolarizados argentinos. International Journal of Psychological Research, 5(2), 40-48.

Llanos, M. (2020) Art, creativity and resilience: resources in the face of the pandemic. Avances $\begin{array}{lllll}\text { en } & \text { psicología, } & \text { Vol.28, } & \mathrm{N}^{\circ} 2, & \text { pp. }\end{array}$ https://doi.org/10.33539/avpsicol.2020.v28n2.2248

Liu, H. \& Chang, C. (2017). Effectiveness of 4Ps Creativity Teaching for College Students: A Systematic Review and Meta-Analysis. Creative Education, (8) 857-869. https://doi.org/10.4236/ce.2017.86062

López, T. (2015). Programación didáctica de Tecnologías en $1^{\circ}$ ESO: Inmersión en la creatividad con la superación de barreras (Trabajo Fin de Máster). Universidad de Valladolid, Segovia, España.

Martínez-Taboas, A. (2020). Pandemias, COVID-19 y Salud Mental: ¿Qué Sabemos Actualmente? Revista Caribeña de Psicología, 4(2), 143-152. https://doi.org/10.37226/rcp.v4i2.4907

Morales, A. (2001). La evaluación en el área de educación visual y plástica de la ESO. https://www.tdx.cat/bitstream/handle/10803/5036/jjma04de16.pdf.PDF?sequence=4\&i sAllowed $=y$

Rhodes, H. (1961). An Analysis of Creativity. Phi Delta Kappan, 42, 305-310.

Romero, J. (2010). Creatividad distribuida y otros apoyos para la educación creadora. Pulso, (33), 87-107.

Sánchez, H. (2017). Arte, creatividad y desarrollo humano. TRADICIÓN, N 17, pp 18-24.

Revista de la Universidad Ricardo Palma.

UCLG (2020) Experiencia de Aprendizaje en Directo: Más allá de la respuesta inmediata a la epidemia de COVID-19. La movilización cultural en la pandemia del COVID-19. https://www.uclg.org/sites/default/files/spa_briefing_culture.pdf 
$t_{\text {tan }}$

HUMANISMO

Unesco (2020) COVID-19 y las industrias culturales y creativas. https://es.unesco.org/sites/default/files/resiliart_-_nota_conceptual_es.pdf

Valqui, R. (2009). La creatividad: conceptos, métodos y aplicaciones. Revista Iberoamericana de Educación, 49(2), 1-11

Vigotsky, L. (2003). Imaginación y creación en la edad Infantil. Nuestra America.

Wallas, G. (1926). The Art of Thought. Kent, Inglaterra: Solis Press 